\title{
Pseudobulbar Affect or Depression - A Clinician's Dilemma
}

\author{
Pranjali Bansal', Shruti Aggarwal ${ }^{2}, \underline{\text { RC jiloha }}{ }^{3}$
}

${ }^{1}$ Senior Resident, ${ }^{2}$ Assistant Professor, ${ }^{3}$ Professor and Head of Department, Department of Psychiatry, Hamdard Institute of Medical Sciences \& Research \& affiliated Hospitals, Jamia Hamdard University, Delhi.

DOI: https://doi.org/10.24321/2581.5822.201914

\section{I $\quad \mathbf{N} \quad \mathbf{F} \quad \mathbf{O}$}

\author{
Corresponding Author: \\ Shruti Aggarwal, Department of Psychiatry, \\ Hamdard Institute of Medical Sciences \\ \& Research \& affiliated Hospitals, Jamia \\ Hamdard University, Delhi. \\ E-mail Id: \\ shruti.aggarwal49@gmail.com \\ Orcid Id: \\ https://orcid.org/0000-0002-7386-6660 \\ How to cite this article: \\ Bansal P, Aggarwal S, Jiloha RC. Pseudobulbar \\ Affect or Depression - A Clinician's Dilemma. J \\ Adv Res Psychol Psychother 2019; 2(3\&4): 35-37. \\ Date of Submission: 2020-01-24 \\ Date of Acceptance: 2020-03-12
}

\section{$\begin{array}{llllllll}\mathbf{A} & \mathbf{B} & \mathbf{S} & \mathbf{T} & \mathbf{R} & \mathbf{A} & \mathbf{C} & \mathbf{T}\end{array}$}

Pseudobulbar affect (PBA) may occur in association with a variety of neurological diseases, and so may be encountered in the setting of traumatic brain injury, stroke, brain tumours and extra pyramidal \& cerebellar disorders. We present a case of 50 years old male, presenting with involuntary crying spells but no significant findings on neuroimaging or other investigations, leading to initial misdiagnosis of depression. Though there are characteristic features that can be recognized clinically for accurate identification of PBA, there needs to be a high level of suspicion and thorough clinical evaluation on the part of clinician for accurate diagnosis of PBA and early intervention.

Keywords: Pseudo Bulbar Affect, Stroke, Mood Disorders, Depression, Anxiety, Involuntary Emotional Expression Disorder (IEED)

\section{Introduction}

Pseudobulbar Affect (PBA) is characterized by the involuntary crying or laughing indicating the disparity between patient's emotional experience with his/ her emotional expression. It may occur in association with a variety of neurological diseases like, amyotrophic lateral sclerosis, multiple sclerosis, traumatic brain injury, stroke, brain tumors \& Alzheimer's disease. ${ }^{1,2}$ Although it is most commonly misidentified as a mood disorder, it is a neurological symptom, presence of which should prompt detailed neurological evaluation.

\section{Case Report}

A tailor of Asian origin in his early fifties, $5^{\text {th }}$ educated, married for 25 years, with well adjusted pre-morbid personality and nil significant past, personal or family history, was brought to the psychiatry outpatient department with complaint of sudden episodes of involuntary crying lasting for few seconds and slurring of speech since 2 days.
Detailed evaluation revealed that 2 days back suddenly while returning back from work he experienced dizziness and sweating lasting for 2-5 minutes following which he came back home on his own. At home while eating, his family members noticed the slurring of speech. Around the same time recurring episodes of uncontrollable, involuntary crying begin, which would last for 2-3 seconds and subside spontaneously. Gradually over one day the frequency of episodes increased and was noticed to be crying every 10-15 minutes, for which patient was brought to the emergency of Hamdard Medical College and research institute, block D, hamdard nagar, new delhi 110062 and got referred to the psychiatry department of the hospital with the possibility of depressive episode.

Patient was admitted in psychiatry ward for further evaluation \& management. Detailed general physical and systemic examination including neurological examination 
revealed no significant abnormality. Mental status examination revealed repeated crying spells in general behaviour with no evidence for the same in either subjective or objective affect assessment. In addition, apart from thought content revealing apprehensions for the crying spells happening against patient's will, rest of the mental status examination revealed no significant psychopathology. Higher metal functions were also found to be within normal limits. Hamilton rating Scale for Depression revealed a score of 5, indicating no significant mood disturbances. Initial investigation including complete hemogram, liver \& renal function test, thyroid function test and lipid profile were also found to be normal.

At this stage, possibility of stroke leading to pseudo bulbar affect was kept and neurology opinion taken. Centre for Neurologic Study-Lability Scale (CNS-LS) ${ }^{3}$ for Pseudobulbar Affect (PBA) was administered. The total score came out to be 17 (on the day of admission), which is suggestive of PBA. Neuroimaging was performed which revealed normal Magnetic resonance imaging Brain (Plain) as well as Electroencephalography.

Though the investigations turned out to be normal, based on clinical picture and in consultation with the neurologist, diagnosis of Pseudo bulbar affect was kept.

As per the treatment guidelines for PBA, patient was started on Selective serotonin reuptake inhibitor Escitalopram 10 $\mathrm{mg}$ along with tapering doses of Clonazepam. With the start of treatment, Scores of CNS-LS scale reduced from 17 to 8 within a span of 3 days, though the slurring of speech persisted.

Patient was reviewed after 1 week post discharge and showed remarkable change in his behaviour and communication. His speech was clearer and episodes of crying reduced significantly and he started going for his job and was advised to continue $10 \mathrm{mg}$ of Escitalopram along with regular follow ups.

\section{Discussion}

Pseudobulbar affect often gets misdiagnosed as mood disorder despite a significant evidence of it having neurological basis. Multiple, interconnected regions of the brain are involved in the appropriate expression of affect. Executive function of the prefrontal cortex, along with the brainstem (the "bulb") is required to generate appropriate affect, such as laughter, crying, and tears. ${ }^{4}$ Although the mechanism is not fully understood, serotonergic and glutamatergic transmission appear to play a major role in affect expression. ${ }^{4}$ For people suffering from a wide variety of neurological conditions affecting these areas, affect is not so natural. As per the diagnostic criteria for PBA given independently by Poeck ${ }^{5}$ and Cummings, ${ }^{6}$ it presents with involuntary and inappropriate expressions of emotion referred to as Pseudobulbar Affect (PBA). There are no mood changes corresponding to the episodes and no sense of relief as the affects are expressed.

In this case, all the investigations including neuroimaging came out to be normal despite the plethora of clinical evidence in favour of diagnosis of PBA. Thus, it reflects and emphasises the age old notion of importance of clinical evaluation rather than relying just on investigations. Moreover, PBA after stroke is prevalent in $11-34 \%$ of patients, but is grossly under diagnosed.7 A good clinical examination along with high index of suspicion can guide the investigations and management in the right direction. This seems to be specifically of significance in developing countries like India where healthcare expenditure by state are still minimal and finances are a matter of significant concern for the patients.

\section{Conclusion}

Pseudobulbar affect is known to occur with various neuropsychiatric disorder and neurological disorders at varying prevalence rate. It is socially and occupationally disabling which requires early recognition and intervention. However, further research is required to understand its pathophysiology.

\section{Ethical Clearance}

It was taken from of Hamdard Medical College and research institute, block D, hamdard nagar, new delhi-110062.

Consent: It was taken from the patient and the attenders.

Source of Funding: Self-funded.

\section{Conflicts of Interest: None}

\section{References}

1. Parvizi J, Arciniegas DB, Bernardini GL, Hoffmann MW, Mohr JP, Rapoport MJ et al. Diagnosis and management of pathological laughter and crying. Mayo Clin Proc 2006; 81(11): 1482-1486. Available from: https:// www.mayoclinicproceedings.org/article/S00256196(11)61255-X/fulltext [PubMed/ Google Scholar].

2. Parvizi J, Coburn KL, Shillcutt SD, Coffey CE, Lauterbach EC, Mendez MF. Neuroanatomy of pathological laughing and crying: a report of the American Neuropsychiatric Association Committee on Research. J Neuropsychiatry Clin Neurosci 2009; 21(1): 75-87. Available from: https://neuro.psychiatryonline.org/ doi/full/10.1176/jnp.2009.21.1.75?url_ver=Z39.882003\&rfr_id=ori\%3Arid\%3Acrossref.org\&rfr_dat $=c r$ pub++Opubmed\& [PubMed/ Google Scholar].

3. Moore SR, Gresham LS, Bromberg MB, Kasarkis EJ, Smith RA. A self report measure of affective lability. J Neurol Neurosurg Psychiatry 1997; 63(1): 89-93. Available from: https://jnnp.bmj.com/content/63/1/89. 
long [PubMed/ Google Scholar].

4. Ahmed A, Simmons Z. Pseudobulbar affect: prevalence and management. Department of neurology, Penn state Hershey medical college, USA. 2013; 9: 483-448. Available from: https://pennstate.pure.elsevier.com/ en/publications/pseudobulbar-affect-prevalence-andmanagement.

5. Poeck K. Pathophysiology of emotional disorders associated with brain damage. In: Vinken PJ, Bruyn GW, editor. Handbook of Clinical Neurology. 1969; 3: 343-367. Available from: https://philpapers.org/rec/ POEPOE.

6. Cummings JL, Arciniegas DB, Brooks BR, Herndon RM, Lauterbach EC, Pioro EP et al. Defining and diagnosing involuntary emotional expression disorder. CNS Spectr 2006; 11(6): 1-7. [PubMed/ Google Scholar].

7. Segamarchi MC, Alet M, Claverie S, Lepera S, Gonzalez LA, Rey R. Pseudobulbar affect in patients with stroke. Neurology 2019; 92(15S): P3.3-028. 